CERN-TH.6413/92

ACT- $2 / 92$

CTP-TAMU-12/92

\title{
Measuring the $W$-hair of String Black Holes
}

\author{
John Ellis and N.E. Mavromatos \\ Theory Division, CERN, CH-1211, Geneva 23, Switzerland \\ and \\ D.V. Nanopoulos \\ Center for Theoretical Physics, Dept. of Physics, \\ Texas A \& M University, College Station, TX 77843-4242, USA \\ and \\ Astroparticle Physics Group \\ Houston Advanced Research Center (HARC), \\ The Woodlands, TX 77381, USA
}

\begin{abstract}
We have argued previously that the infinitely many gauge symmetries of string theory provide an infinite set of conserved (gauge) quantum numbers ( $W$-hair) which characterise black hole states and maintain quantum coherence. Here we study ways of measuring the $W$-hair of spherically-symmetric four-dimensional objects with event horizons, treated as effectively two-dimensional string black holes. Measurements can be done either through the s-wave scattering of light particles off the string black-hole background, or through interference experiments of Aharonov-Bohm type. In the first type of measurement, selection rules restrict the number of particles emitted by the black hole, while in the second method the wave-functions of fundamental strings scattered off the black hole have phases that can be measured in appropriate interference experiments.
\end{abstract}

CERN-TH.6413/92

$\mathrm{ACT}-2 / 92$

CTP-TAMU-12/92

February 1992 


\section{Introduction}

The reconciliation of general relativity with quantum mechanics is one of the key problems in physics. One of its many facets is the quantisation of gravitational effects in a flat space-time background, including the calculability of perturbation theory, which should be finite or at least renormalisable. Another facet is the problem of quantisation in non-flat space-times that are solutions of the gravitational field equations. Still another is the full non-perturbative treatment of fluctuations in space-time. And so on...

Conventional point-like quantum field theory has been unable to resolve any of these problems, whilst string theory is an ambitious candidate for a framework in which they can all be resolved. Indeed, it has now been established that string perturbation theory in a flat space-time background is finite [1]. On the other hand, the full non-perturbative treatment of fluctuations in space-time requires the development of a fully-fledged string field theory, which so far only exists for simplified toy models of string gravity coupled to matter [2, 3]. In a series of recent papers [ [ , 5, 6, 7], we have been studying the intermediate problem of quantum theory in a non-flat space-time background.

Specifically, we have been investigating whether conventional quantum coherence can be maintained in a black hole background in string theory. Semiclassical arguments [8] in conventional point-like quantum field theory indicate that macroscopic black holes behave like mixed, thermal states, which has motivated suggestions 9] that quantum coherence cannot be maintained at a fundamental level when microscopic non-perturbative fluctuations in space-time are taken into account. The root of this problem with quantum coherence is the observation that the apparent entropy $S$ of a black hole is proportional to the area of its event horizon [8, 10]: $S \propto A$, which is in turn $\propto M^{2}$ for an uncharged axisymmetric black hole. Thus the apparent entropy of a black hole is unbounded, whereas any point-like quantum field theory has only a finite set of quantum numbers. These are insufficient to encode all the information carried by matter that collapses gravitationally, and thereby distinguish all the states of a black hole, which must therefore be described by a mixed state.

However, we have argued [4, 5] that there are infinitely many gauge symmetries in string theory, which lead to an infinity of conserved quantities, "W-hair", that maintain quantum coherence in the presence of a black hole. First demonstrated in a two-dimensional model, this argument applies also to four-dimensional black holes, as has been shown explicitly in the spherically-symmetric case of physical interest [7]. It has been shown in general [6] that, within the context of string theory, black hole decay can be calculated as a conventional quantum-mechanical process which does not involve thermal or other mixed states, and the number of pure solitonic states of a stringy black hole has been estimated [7] and shown to correspond to the number of black hole states found previously by semi-classical 
field-theoretic arguments [8, 10]. Thus the entropy of a four-dimensional black hole is only apparent, and can in principle be reduced to zero by measurements of the intrinsically stringy $W$-hair quantum numbers of these solitonic states.

The question then arises, how could one imagine measuring these quantum numbers in practice? In this paper we propose two types of possible measurement. One is via selection rules for the scattering of light particles off stringy black holes, and the other is an infinite set of stringy Aharonov-Bohm effects.

The concept of the first type of measurement is very similar to that of $\pi$-nucleon scattering in the Skyrme model [11]. In that case the $\Delta(1232) 3-3$ resonance, for example, is a higher spin (and isospin) soliton which can be excited if the $\pi$ energy is resonant, and its decay satisfies certain selection rules. This picture has been extended to higher resonances, with soliton calculations reproducing well the phase shifts in different partial waves [12] and general selection rules derived [13]. In our case, there is an infinite set of black hole soliton states, classified by the quadratic Casimir and 'magnetic' quantum numbers of an internal symmetry group, which are excited at calculable energies and decay into distinctive numbers of light finalstate particles. These results are derived in the limiting case of a flat space-time background that represents the end-point of black hole decay, and then in the generic (potentially macroscopic) black hole case. The key rôle of the $s$-wave dynamics and the particle production selection rules are reminiscent of the Callan-Rubakov [14] process in scattering off a monopole.

The second type of measurement involves the characteristic inteferences between states propagating in the neighbourhood of, and far from, a black hole. Since there are infinitely many massive string states which can be scattered in this way, there is an infinity of such possible measurements, albeit with certain practical difficulties in the case of a macroscopic black hole.

The layout of this paper is as follows. In section 2 we review the infinite set of stringy $W$-symmetries on the world-sheet and their elevation to physical space-time symmetries. In section 3 we derive the selection rules for scattering in a flat spacetime background [15], and extend these to the generic black hole case in section 4 . Section 5 presents the generalised Aharonov-Bohm measurements, and we discuss some outstanding issues in section 6 . 


\section{W-symmetries on the world-sheet and in phys- ical space-times}

We begin our study with a brief review of the $W$-symmetries possessed by string theories in two-dimensional space-time, and hence also string theories in sphericallysymmetric four-dimensional space-time.

The first notion of a target space-time $W$-symmetry was presented by Avan and Jevicki [16] in the context of the collective field representation of the $c=1$ matrix model [2]. The latter is nothing other than string field theory for the only propagating degree of freedom of the two-dimensional strings, the so-called 'tachyon', which is actually massless in such theories. The existence of an infinite-dimensional Cartan subalgebra of conserved charges was demonstrated. Subsequently, it was suggested

[四] that these symmetries could be understood as 'hidden' gauge symmetries [17] of the underlying string theory, associated with the higher excited string states, which in two dimensions are non-propagating and have definite values of energy and momentum. These states had been known to exist in intermediate channels of tachyon scattering amplitudes in matrix models [18], in Das-Jevicki theory [19], and in $c=1$ Liouville theory [20], but their physical significance had not been realised at the time. It is by now clear that these modes are essential for the perturbative unitarity of flat-space string scattering matrix [21]. In fact, it is through the usual factorisation via the operator product expansion (OPE) of vertex operators that these discrete states are produced as intermediate states in tachyon scattering amplitudes. Soon after the suggestion of ref. [4] on the association of $W$-symmetries with higherlevel string states, Moore and Seiberg [22] constructed explicitly a $W_{1+\infty}$ algebra of symmetries in the fermionic representation of $c=1$ matrix models, and showed its connection with the higher-spin (discrete) string modes. In view of the target-space interpretation of the model as a field theory of the tachyon field [2], these charges characterise the flat string background in two dimensions, or the $s$-wave sector of dimensionally-reduced four-dimensional strings [7].

Although the target-space interpretation of these symmetries was evident by construction, it is still useful to understand their origin as world-sheet symmetries, which would then be elevated to target-space ones in the usual way, via induced canonical deformations of the corresponding conformal field theory [23.

Let us be more precise. String theory in first-quantised form is formulated as a theory on the world-sheet. Hence, at a superficial level the only apparent symmetries are those on the world-sheet. One is then interested in knowing under which conditions such symmetries could be elevated into symmetries of the physical spacetime. Let $h=\int d \sigma j(\sigma)$ be a conserved charge of a current $j(\sigma)$ generating such a symmetry. This implies the invariance of the Fradkin-Tseytlin generating function for amplitudes under an appropriate change of the $\sigma$-model fields (target-space co- 
ordinates) that corresponds to the symmetry in question [17]. The parameters of the transformation may, and in fact do in our case [5], have an explicit dependence on world-sheet coordinates. This makes this formalism unwieldy for getting nonperturbative results in some closed form. As an alternative, Evans and Ovrut [23] suggested the study of hidden symmetries through the induced deformations on the stress-tensor of the conformal field theory in a certain background $\{\mathrm{g}\}$. If $T_{g}\left(\bar{T}_{g}\right)$ denotes the holomorphic (antiholomorphic) part of the stress tensor of the $\sigma$-model, then the induced (infinitesimal) deformation is $\delta T_{g}=i\left[h, T_{g}\right]$. The deformation is a symmetry of the physical (target) space-time if

$$
\delta T_{g}=T_{g+\delta g}-T_{g}
$$

for some induced transformation of the couplings: $g \rightarrow g+\delta g$. A deformation is said to be canonical if 23

$$
\delta T_{g}=\Phi_{(1,1)}
$$

where $\Phi_{(1,1)}$ is a primary field of dimension $(1,1)$. Thus conformal invariance is automatically satisfied for canonical deformations. Due to the completeness of the set of $(1,1)$ vertex operators in string theory, it is also evident, in view of (1) , that a canonical deformation is also a symmetry of target space-time. An important comment is that, if the current generator of the world-sheet symmetry is an operator of conformal dimension $(1,0)$ or $(0,1)$, then the induced deformation has conformal dimension $(1,1)$ and, hence, generates a target space symmetry of the background. This is precisely what happens in the case of two-dimensional string theory in flat space-times 24]. There is an infinity of $(1,0)$ or $(0,1)$ world-sheet currents, constructed out of states of non-standard ghost number, which generate $W$-symmetries on the world-sheet that can be lifted [5] to $W$-symmetries of the physical spacetime of two-dimensional string theory, formulated on flat backgrounds. These symmetries have the properties that they leave invariant under target time evolution the two-dimensional tachyon phase-space, which, in view of [2], is equivalent to a matrix-model phase space [24]. Following indications about background independence of the Das-Jevicki string feld theory [2, 25, 5], one might expect [5] that these $W$-symmetries, or some appropriate deformations of them, would persist in highly-curved string backgrounds such as black holes [26, 27]. The phase-space-areapreserving character of the symmetries, then, would guarantee the maintenance of quantum coherence even during extreme physical processes like black hole evaporation/decay, for the reasons argued in [5].

To check these considerations explicitly for curved space-times seems a difficult task. The weak-field expansion methods that are used in extracting the $\beta$-functions of $\sigma$-model perturbation theory might not prove sufficient for getting exact information about complicated backgrounds such as black holes. On the other hand, already from critical string theory we know of cases where exact conformal field theories have been used to circumvent the patterns of perturbation theory around 
complicated string ground states, like Calabi-Yau compactified spaces. Gepner's construction 28] of tensoring superconformal field theories has provided us with non-perturbative information about exact solutions of string theory in the form of Calabi-Yau Ricci-flat spaces, which seemed not to correspond to solutions of perturbative $\beta$-function equations [29]. In Gepner's construction an important method was the use of Fateev-Zamolodchikov parafermions to represent the pertinent $N=2$ superconformal algebras. It is in this representation that extra selection rules, not known previously, have been found for correlation functions in Calabi-Yau backgrounds [30.

Motivated by these results in critical string theory, it was natural to ask whether similar constructions of exact conformal field theories can be found in the case of string black hole backgrounds. This question was answered positively by Witten [27], who has shown that it is possible to describe the interior of the horizon of a black hole in two-dimensional space-time using a coset $\frac{S L_{k}(2, R)}{G}$ Wess-Zumino(WZ) model, with $G=S O(1,1)\left(G=U(1)_{\text {compact }}\right)$ for Minkowskian (Euclidean) black holes. The important point is that this is the first time that a finite theory, like the WZ model, is used to describe a target space-time singularity 7 . And it is this kind of singularity that thwarted earlier attempts to describe black holes, or in general singular objects surrounded by event horizons, in a way consistent with quantum mechanics [8]. Conformal field theory provides, as we shall see in section 3, the consistent construction of a scattering matrix to describe scattering of propagating string states (tachyons) off the black hole background. This is to be contrasted with the situation in point-like field theories, where such a scattering matrix was argued not to exist 8, 32.

In parallel to the parafermion representation of Gepner's spaces, one could expect a similar construction here, as was indeed shown in 33. Any state that admits an expansion in terms of an $S L(2, R)$ current-algebra basis can be represented as a highest weight $N=2$ superconformal state, which admits a parafermion realisation [34]. In bosonised parafermion language, an $S L(2, R)$ algebra is realised by three bosons. To describe a coset requires factoring out one of them, and one is left with a two-boson realisation of the coset model. In complex notation, let $\phi(z), \bar{\phi}(z)$ be the two bosons, with $j=\partial_{z} \phi, \bar{j}=\bar{\partial}_{z} \phi$ the corresponding currents. The parafermion currents are expressed as

$$
\psi_{+}=\bar{j} e^{\phi+\bar{\phi}}, \psi_{-}=j e^{-(\phi+\bar{\phi})}
$$

\footnotetext{
${ }^{1}$ However, it should be stressed that the conformal field theories in question are not a mere ansatz for describing known local field theory objects like traditional Schwarzschild solutions of Einstein's equations. On the contrary, they serve to demonstrate the fact that objects resembling the space-time singularity structure of spherically-symmetric non-rotating four-dimensional black holes [7] appear as exact solutions of subcritical string theory [31]. This is similar in spirit to Gepner's demonstration that Calabi-Yau (Ricci-flat) spaces are exact (non-perturbative) solutions of critical string theory.
} 
The $W$-symmetry structure is revealed by looking at the classical OPE between parafermion currents $\psi_{+}(z) \psi_{-}(w)$, for $z \rightarrow w$ [35]. Expanding in powers of $\epsilon \equiv z-w$ and keeping all powers of $\epsilon$

$$
\psi_{+}(z) \psi_{-}(z+\epsilon)=\sum_{r=0}^{\infty} u_{r}(z) \frac{\epsilon^{r}}{r}
$$

one discovers a world-sheet $W$-algebra, generated by the commutation relations of the $u_{r}$ [36, 37]. Parenthetically, but importantly, we notice that the coefficients $u_{r}$ are world-sheet currents of conformal spin $r+1$ [37], which appear as expansion coefficients in the pseudo-differential operator $L=\partial_{z}+\sum_{r=0}^{\infty} u_{r}\left(\partial_{z}\right)^{-r-1}$, defining the so-called $K P$ Hamiltonian basis. In this sense the appearance of $W$-symmetries is related to the $K P$ hierarchy, which might be suggestive of new ways of approaching $c=1$ string theory [37]. In the coset model, the symmetry algebra generated by the currents $u_{r}$ is related to the second Hamiltonian structure of the $K P$ hierarchy [36]. It is actually a non-linear deformation [37], $\hat{W}_{\infty}$, of the centerless $w_{\infty}$ algebra of Bakas 38. We shall come back to this point in section 4. Quantisation of the model does not simply require normal ordering these OPEs, but a redefinition of the currents [36] so as to ensure the closure of the quantum $\hat{W}$-algebra. Details of the construction can be found in [36].

An infinite set of commuting quantum $W$-charges is constructed as world-sheet spatial integrals of the currents $u_{r}$ (or rather an appropriate redefinition, $W_{r}$, in the notation of [37, 35]), with integer conformal spin $s \geq 2$ [36]

$$
\left[\int d z W_{s}(z), \int d w W_{s^{\prime}}(w)\right]=0 ; s, s^{\prime} \geq 2 .
$$

The charge $W_{2}$ coincides with the Hamiltonian of the model, or, in the case of closed strings, with the holomorphic part of the $L_{0}$ Virasoro operator. Notice also that spin-one objects are not included, by construction [35, 36], in this set and so the deformed algebra is not of $W_{1+\infty}$ type (which is generated by integer conformal spins $s \geq 1$ ). The $W_{\infty}$ algebra is known [37] to be a subalgebra of $W_{1+\infty}$. However, it is the $w_{\infty}$ algebra that has a well-known geometrical interpretation as a phasespace area preserving symmetry [38], and so for our purposes [t. it is sufficient to concentrate on $w_{\infty}$ and its quantum deformation $\hat{W}_{\infty}$.

The above constructions are valid for coset WZ models whose level parameter $k \geq 2$ [35, 36]. The model with $k=\frac{9}{4}$ admits an interpretation as a critical string theory propagating in a black hole background [27]. The interesting point is therefore the lifting of this enormous world-sheet symmetry to a physical gauge symmetry

\footnotetext{
${ }^{2}$ It should be mentioned, though, that in view of the matrix-model result [22], one might expect the actual target space-time symmetry of the two-dimensional string theory to be bigger, allowing for symmetries generated by conformal spin-one currents. This would include the canonical deformations mentioned above. Of course, one cannot exclude the possibility that the flat spacetime symmetries are larger than those of the black hole background.
} 
of two-dimensional strings. Due to the higher conformal spins of the generating currents, it turns out that the induced deformations about the pertinent background are not in general canonical. As argued in [39] recently, canonical deformations are only a part of the enormous infinite set of target gauge symmetries. The requirement that a field be $(1,1)$ implies usually an equation of motion and some gauge conditions (constraints), for higher-spin states. The most general set of deformations discussed in [39] relaxes the requirement of gauge fixing, and hence one works in arbitrary gauges, so the only remaining constraint is conformal invariance. The main conclusion then is that any symmetry on the world-sheet, generated by a current of arbitrary conformal spin, can be viewed as generating a symmetry of target spacetime, provided translational invariance is maintained on the world-sheet. Therefore the corresponding charge operator should commute with $L_{0}-\bar{L}_{0}$ :

$$
\{h\}:\left[h, L_{0}-\bar{L}_{0}\right]=0
$$

The cost one pays is the introduction of auxiliary fields that are pure gauge artifacts introduced to count correctly degrees of freedom [39]. In the two-dimensional case, as we shall discuss in the next section in some detail, the existence of discrete modes in higher spin levels, which are associated with the target $W$-symmetries, emerges precisely from a relaxation of constraints and/or gauge conditions which occurs at particular values of energy and momentum [20]. Moreover, the worldsheet charges (5) do satisfy (6) by construction, and therefore they are responsible for the generation of string gauge symmetries in physical space-times. In the context of first-quantised string theory, these symmetries will be expressed through complicated redefinitions of the $\sigma$-model fields [5]. From the commutation relations (5) it becomes clear that the induced deformations vanish when integrated over the world-sheet. This should be intuitively expected. The ordinary target-space general coordinate transformations are in fact total derivative effects on the world-sheet, being mainly responsible for the difference of local from global conformal invariance (c.f. the difference of $\beta$-functions from $\bar{\beta}$-functions is expressed as a general coordinate transformation [40]). Also, from a glance at the commutation relations of the charges (5) with the string level operator in the corresponding string theory, it becomes evident that for many of them there is a non-vanishing (and rather complicated) result, thereby implying a mixing of string levels, as expected for a stringy gauge symmetry transformation.

\section{Selection rules for scattering in flat space-time}

Here we review briefly the situation in the flat space-time $c=1$ string. The latter is interpreted both as the asymptotic (spatial infinity) form of the black hole space-time as well as the end-point (temporal infinity) of perturbative black hole evaporation [27, 6]. We shall put emphasis mainly on the physics of this background rather than the mathematical details. The important point is that the $c=1$ conformal matter theory coupled to Liouville gravity admits a space-time interpretation with the Liouville field $\phi$ being considered as the spatial coordinate, and the 
Feygin-Fuchs representation $\chi$ of the matter field as the temporal one ${ }^{3}$. Viewed as a flat space-time string theory, the $c=1$ Liouville-matter system resembles that of an ordinary subcritical string theory with an anomaly $Q=\sqrt{\frac{c-25}{3}}=2 \sqrt{2}$ in the Liouville sector, but with imaginary "Liouville energies" [20]. For example, the propagating massless modes of the system, called misleadingly 'tachyons', turn out to be described by $(1,1)$ vertex operators of the form:

$$
V^{T}=e^{i k_{\mu} X^{\mu}}
$$

where $X^{\mu}=(\phi, \chi)$ and $k^{\mu}=(i \epsilon(p), p)$. The requirement that the operator $V^{T}$ be a $(1,1)$ field implies an equation of motion $k^{\mu}(k+Q)_{\mu}=-2$ (with $Q_{\mu}=(i Q, 0)$ ) for the tachyon field, which is the analogue of the 'on-shell condition' in ordinary string models. The solution requires that the Liouville energies are not independent of the matter momenta $\mathrm{p}$, but rather:

$$
\epsilon(p)=-\sqrt{2}+\alpha p
$$

where $\alpha= \pm 1$. Notice that $p$ denotes the vector and not the magnitude of the matter momentum. In view of the space-time interpretation of the matter momentum as energy, and the Liouville energy as (radial) momentum, the two signs in (8) indicate the two possible directions (outgoing and ingoing) of the radial momentum (this is also true in the case of spherically-symmetric four-dimensional space-times). As remarked in 41], in Liouville-gravity only states with momentum $p_{\phi} \equiv \epsilon \geq-\sqrt{2}$ are kept, since these are the only ones that have well-behaved asymptotic behaviour in the limit where the Liouville field, assumed as a free field, approaches $-\infty$. Hence in (8) $\alpha p>0$ for both signs of $p$, thereby fixing a sign for $\alpha$ for a fixed sign of $p$. This defines uniquely the concept of an 'ingoing' or 'outgoing' particle in this picture.

In addition to the tachyons, which are propagating light particles, the $c=1$ string theory has an infinity of discrete (higher-spin) excited states with definite energy and momentum. These states make their appearance in the subleading terms of OPE of tachyon vertex operators [18, 21]. Their presence is essential for perturbative unitarity of the scattering matrix $S$ of the $c=1$ string theory. The discrete states do not appear as external on-shell states due to their quasi-topological nature; they are rather soliton-like states that appear as exchanged states in amplitudes of tachyons, and can be found by factorisation [15]. There appear to be two branches of discrete states [15], one corresponding to the degeneration of gauge transformations associated with the given string level, and the other to the relaxation of the constraints imposed on the polarisation tensors of the states for generic momenta. The selection rules discussed in this section refer to the first branch. The reason is simple. In flat space-time strings these states are singular gauge transformations, and so can be

\footnotetext{
${ }^{3} \mathrm{An}$ alternative interpretation of the model as describing physics in a Euclideanised twodimensional universe, with the Liouville field being the Euclidean time, seems not to be compatible with the interpretation of the model as an asymptotic form of the black hole solution, where the Liouville field clearly plays the rôle of the radial (spatial) coordinate [27].
} 
regarded as physical gauge particle states that interact with the massless (propagating) degrees of freedom, the tachyons. The other branch seems to play an important rôle in highly-curved backgrounds. For instance, at level 1 the states of the second branch have momentum $k_{\mu}=-Q_{\mu}$, and they constitute the excitations of the limiting (vanishing mass) black hole solution [26, 4]. So the natural question concerns the physical independence of these branches of string states. As we mentioned above, from the point of view of free-field Liouville gravity, a state with momentum $p_{\phi} \equiv \epsilon<-\frac{Q}{2}$ is excluded [41], due to bad asymptotic behaviour in the limit where the Liouville field $\phi \rightarrow-\infty$. The situation of course is not clear if boundaries are imposed on Liouville space from below, which appears necessary due to the rôle of the Liouville field as a cut-off on the world-sheet [42, 43]. We shall resolve such issues in the context of the next section. As we shall discuss [44], the other branch seems to correspond to equivalent $S L(2, R)$-isospin representations, and again the discrete states are the ones that look like singular gauge transformations.

Before going into details of the selection rules, it is essential to discuss some features of the tachyon amplitudes in this string model. The most important (and probably mysterious) of them is the fact that the only non-vanishing amplitudes for generic values of the momenta of external tachyons are the ones with signatures $+++\ldots+-$ or $---\ldots-+$, where the sign is that of $\alpha$ in (8). From a physical point of view this is very strange, since it implies that only amplitudes with either one incoming or one outgoing particle are non-vanishing for generic tachyon momenta. The remaining amplitudes vanish modulo contributions that are $\delta$-functions of the momenta [15]. The explanation we offer is the target space $W$-symmetries. There exist Ward identities in target space-time, which are the continuum analogues of corresponding identities found in the $c=1$ matrix model [45]. We argue that these are responsible for the above selection rules for particle scattering in subcritical string theory. Given that the $W$-symmetries have their origin on the world-sheet, these Ward identities are similar in nature to those found in critical string theory [17, and correspond to redefinitions of the target space coordinates. As we have already mentioned, a similar in nature (but technically more complicated ) redefinition of the $\sigma$-model fields operates here as well [5]. These issues certainly deserve more rigorous treatments than our simple arguments, and we hope to come back to them in a future publication 46 .

In addition to these strange selection rules, there are additional ones for resonant amplitudes of the form $+++\ldots+-$ (or its mirror), involving resonances of discrete states, which restrict the number of light particles interacting with them. For instance, consider the $N$-tachyon amplitude [20]

$$
A_{++++\ldots+-}\left(p_{1}, \ldots, p_{N-1}\right)=\frac{1}{(N-3) !} \frac{\Gamma\left(1-2 p_{1}\right)}{\Gamma\left(2 p_{1}\right)} \ldots \frac{\Gamma\left(1-2 p_{N-1}\right)}{\Gamma\left(2 p_{N-1}\right)}
$$

It is worth pointing out that this is only the 'resonant' part of the amplitude as far as Liouville energies are concerned. In Liouville theory coupled to matter, due 
to the peculiar Liouville dressing of matter operators, there is not in general conservation of Liouville energies. However, the amplitudes have 'resonant' forms [20], for the description of which free-field methods are applicable. This means that the boundaries in Liouville field $\phi$-space are ignored and the Liouville field is treated as a free boson. All the physical amplitudes are assumed to be the residues of Liouville energy conservation poles, and hence both energies and momenta are assumed conserved, as in ordinary string theory, implying $S L(2, R)$ invariance in the usual Koba-Nielsen form. The kinematics then implies:

$$
\begin{aligned}
\sum_{i=1}^{N-1} p_{i} & =\frac{N-2}{\sqrt{2}} \\
p_{N} & =-\frac{N-2}{\sqrt{2}}
\end{aligned}
$$

where one should be careful to include the anomaly $Q$ in the Liouville energy conservation law. Combinining then energy and momentum conservation one finds that one of the momenta is completely determined, for instance $p_{N}$.

The amplitude (9) has 'leg' poles at momenta:

$$
p=\frac{n}{2} ;\{n\}=Z^{+}
$$

In usual string theory, poles in external particle states are explained by factorising the amplitude using the OPEs of two vertex operators approaching each other on the world-sheet. A similar thing happens here [21]. When a pole occurs, say in the $(1, N)$ particle-channel, then the conservation equations imply restrictions on the number $N$ for a given exchanged discrete state.

The discussion is facilitated if one uses a more convenient set of quantum numbers to classify the discrete states, rather than merely their spin and string level number as in ordinary (critical) string theories. Due to the low dimensionality of the target space-time, it turns out that the string states can be classified by assembling them in $S U(2)$ multiplets [15]. At this point, it is worth making a digression which will be useful for gaining some intuition about the classification of states around more complicated backgrounds like black holes, which will be discussed in the next section.

The value of the central charge of the conformal field theory plays a crucial rôle in the sort of representation one uses to represent the string states. Fateev and Zamolodchikov have shown that for $c<3$ unitary CFT one could convert the parafermion algebra used to describe the states into a current algebra of $S U(2)$. On the other hand, for $c>3$ one has to use $S L(2, R)$ non-compact current algebras [33]. We shall come back to these issues in section 4 . For our purposes, the states of the $c=1$ flat space-time string theory can be assembled in $S U(2)$ representations, 
characterised by two quantum numbers, the $\mathrm{SU}(2)$ isospin $j=\frac{n}{2}, n$ integer, and the magnetic number (its third component) $m=-j,-j+1, \ldots, j$. Following Klebanov and Polyakov [15], and using their normalisation in which the two-momentum is rescaled by a factor of $\sqrt{2}$, the conformal invariance condition for an operator corresponding to a discrete state after Liouville dressing $e^{\epsilon(j) \phi}$ reads in terms of $j, m$ as:

$$
j^{2}-\epsilon(\epsilon+2)=1-N
$$

for a level $N$ state. From this and the analogy with string theory, where the lefthand part of (12) is just the two-momentum squared, one finds the analogy between the two pictures:

$$
\begin{array}{r}
p \rightarrow m \\
\epsilon^{ \pm} \rightarrow-1 \pm j
\end{array}
$$

The two signs correspond to two different branches of discrete states, corresponding either to singular gauge transformations or to a relaxation of constraints imposed (for generic momenta) on the polarisation tensors of higher excited states. The discrete states that lead to selection rules in flat space-times are the ones that correspond to singular gauge transformations, which are those with the positive branch in (13). For more details we refer the reader to the literature [15].

The selection rules on amplitudes (9) follow immediately upon factorisation and use of (11). At each trilinear OPE vertex there is exact energy-momentum conservation without the screening charge $Q$. This is attributed as a $Q$-insertion to the remaining part of the diagram. Using the analogy (13) we get $2 p_{1}=j+m+1=$ $2 j-(N-4)$. From (11) we get the following selection rules for the emission of the $(j, m)$ state [15]:

$$
\begin{aligned}
& j=m+N-3 \\
& j \geq \frac{N-3}{2}
\end{aligned}
$$

where $N-2$ is the number of particles interacting with the discrete state $(j, m)$. From (14) we observe that in a scattering resonant process where an intermediate topological state $(j, m)$ is excited, the number of emitted particles is restricted, or vice versa, in a process where $N$ particles are scattered, the quantum numbers of the internal states are restricted according to (14). In the space-time interpretation of this theory as a spatially-asymptotic form of a black hole, this kind of selection rule yields some information about the target-space gauge symmetries characterising the black hole configurations ( $W$-hair). In view of the group-theoretic equivalence of the two branches of discrete states in that model, the corresponding selection rules can be considered physically complete. We shall return to these issues in the next section. At present, we remind the reader that the states of the $c=1$ string form a $W$-algebra that admits a phase-space area-preserving interpretation [15, 24]. The 
selection rules given above, both the vanishing of $+++\ldots--\ldots-$ amplitudes as well as the rules (14), are a manifestation of this symmetry. In purely flat space-time backgrounds, of course, the resulting charges are trivial. It is when one considers more complicated string vacua, as we shall do next, that the corresponding selection rules give information about the gauge charges that characterise, say, the black hole. In that sense, the rules (14) are part of the asymptotic form of selection rules for scattering light particles off the black hole background by excitation of black hole topological (internal) degrees of freedom.

\section{Selection rules for scattering off black holes}

The above situation changes significantly when we describe highly-curved string backgrounds such as black holes. Witten showed [27] that there is an exact description of such objects as coset WZ models for a given value, $k=\frac{9}{4}$, of the level parameter. In contrast to Liouville gravity, for this value of $k$ the conformal field theory per se has central charge 26 to compensate the ghost contributions. Therefore, in view of the Fateev-Zamolodchikov result [33] one expects the compact $S U(2)$ picture for the classification of states to break down. Indeed, as discussed in [33], the appropriate classification can be made via $S L(2, R)$ isospin representations, as we mentioned in section 2. The contact with Liouville gravity, discussed in the previous section, is made only asymptotically, either at spatial infinity or in the limiting (zeromass) black hole case [4, 26]. However, it should be stressed that the coset string model seems to be characterised by more states than the flat $c=1$ Liouville model. A rather complete discussion of the spectrum is given in 44 where we refer the reader for mathematical details. Here we shall concentrate on some aspects of that work relevant for obtaining selection rules revealing the underlying $W$-symmetry structure of the target space [5, 35, 36]. We should emphasize that there are differences [44, 6] between Minkowskian and Euclidean black holes, viewed as coset conformal models. One is not obtained from the other via a simple Wick rotation. In particular, the spectrum of physical states is different. There are more states in the Euclidean case, which is natural from the point of view of the target space-time interpretation, due to the compactification of the (target) 'time' coordinate. Some aspects do transcribe simply, though, from Euclidean to Minkowski space, and it is in this sense that it is useful sometimes to formulate the problem in Euclidean space, where it is easier mathematically, and then pass to the Lorentzian signature formalism. Here we shall follow the latter method; the simple Wick rotation is sufficient for the selection rules.

Following Distler and Nelson [44], we characterise the states in the coset model

$\frac{S L_{\frac{9}{4}}(2, R)}{U(1)}$ by two quantum numbers, the $S L(2, R)$ isospin $j$ and its third component $m$, the latter being defined by the diagonalisation of the generator of the $U(1)$ current algebra. The requirement for a state to be a Kac-Moody primary leads to 
the 'on-shell' conditions 44]

$$
\left(L_{0}-1\right)\left|j, m>=\left(-4 j(j+1)+\left(\frac{2 m}{3}\right)^{2}-1\right)\right| j, m>=0
$$

which can be solved to yield

$$
j=-\frac{1}{2} \pm \frac{1}{3} m
$$

Higher-level string states, corresponding to non-zero 'oscillator number' $N$, also exist, but at discrete values of $j$ and $m$. We shall come back to them later on. The lowest oscillator number states are just the 'tachyons' of the two-dimensional string theory. From the asymptotic connection with flat-space two-dimensional strings one can make the following analogy flbetween the quantum numbers $(j, m)$ and the plane wave two-momentum $\left(i \epsilon_{\phi}\left(p_{x}\right), p_{x}\right)$

$$
\begin{array}{r}
j \rightarrow \frac{\epsilon_{\phi}}{2 \sqrt{2}} \\
m \rightarrow \frac{3 p_{x}}{2 \sqrt{2}}
\end{array}
$$

where $p_{x}$ denotes the momentum corresponding to the matter part of the conventional $c=1$ Liouville-matter system. In the space-time black hole interpretation of the model [27, the Liouville field would play the rôle of (spatial) radial momentum and $p_{x}$ the rôle of energy. The two signs therefore in (16) indicate the outgoing or ingoing direction of the (asymptotically plane) wave front.

It is important to notice that $S L(2, R)$ representations are classified by the Casimir $j(j+1)$ rather then $j$ alone, so the physically inequivalent representations are restricted (for the Euclidean model where $j$ is real) to

$$
j \geq-\frac{1}{2}
$$

From the analogy $(17)$ we then recover the restriction $\epsilon_{\phi} \geq-\sqrt{2}$, familiar from $c=1$ Liouville gravity as discussed in section 3. It is this group-theoretic equivalence of representations that leads to the conclusion that the only physically inequivalent states are those corresponding to singular gauge transformations. This is consistent with the asymptotic connection of the model to the flat $c=1$ string theory, as well as the fact that it is this branch of discrete states that seems to be responsible for the $W$-symmetry structure [15, 4, 36, 35] which is common to both theories.

\footnotetext{
${ }^{4}$ It should be stressed that this is only an analogy, or an approximation at spatial infinity perhaps. The states of the coset model cannot be represented as plane waves in target space, due to the highly-curved space-time structures.
} 
From the asymptotic Liouville-like intepretation it is also clear that similar selection rules to those applying in the previous section also apply here. For instance, the vanishing, for generic momenta, of tachyon amplitudes of signatures $+++\ldots--\ldots-$ (or $--\ldots++\ldots+$ ) also occurs here, and can be thought of, along with the additional selection rules referring to amplitudes with $++\ldots-($ or $-++\ldots+)$ to be discussed below, as a manifestation of the $W$-symmetry characterising the target space of the coset model. At this point it should be mentioned that the very fact that this symmetry is of similar nature as that of the $c=1$ Liouville-matter system is an additional indication of an underlying 'hidden' gauge symmetry structure that describes evaporation/decay of the black hole space-time and its evolution to reach asymptotically (in time) flat target spaces. We shall briefly comment on this important issue in section 6 .

The selection rules for the non-vanishing amplitudes $+++\ldots-$ (or its mirror) are obtained in a similar way as those of section 3. Some remarks are in order, however. The first concerns 'energy-momentum' conservation in this context, which would again imply that from the point of view of Liouville-theory the correlation functions of the coset model would reproduce only the resonant parts of the amplitudes, where free-field methods are applicable. That this is indeed the case becomes evident from the free-boson realisation of the coset model [35]. The generic vertex operator $V_{m}^{j}$ of the (Euclidean) coset theory, corresponding to a state characterised by the two quantum numbers $(j, m)$, is represented as

$$
V_{m}^{j}=e^{\frac{8 i m}{9} \chi} Z_{m}^{j}
$$

where $Z_{m}^{j}$ is a primary of the $N=2$ superconformal theory used to represent the $S L(2, R)$ current algebra [33], and $\chi$ is a free boson. It is then clear that in correlation functions for operators of the form (19) there is conservation of the $U(1)$-charge $\propto m$, as a result of the boson zero-mode integration. In view of the on-shell condition (16), this also implies $j$-isospin conservation. The plane wave analogy is then transcribed into energy-momentum conservation. It is important, however, to notice that the space-time interpretation of the model requires the presence of a Liouville screening operator $\epsilon_{\phi}=-\sqrt{2}$ in correlation functions. This means that a $Q$-anomaly insertion term will always be present in Liouville energy $\left(\epsilon_{\phi}\right)$ conservation laws.

We now briefly review the spectrum of the Euclidean coset theory found by Distler and Nelson 44] ( $r, s$ are integers):

$$
\begin{aligned}
\tilde{D}^{ \pm}-\text {series } & : \quad\left(\epsilon_{\phi}, p_{x}\right)=\left(\frac{1}{2 \sqrt{2}}(2 s+4 r-5), \pm \frac{2 s-4 r-1}{2 \sqrt{2}}\right) \\
D^{\mp}-\text { series } & : \quad\left(\epsilon_{\phi}, p_{x}\right)=\left(\frac{1}{\sqrt{2}}(s+2 r-3), \pm \frac{s-2 r+1}{\sqrt{2}}\right) \\
C-\text { series } & : \quad\left(\epsilon_{\phi}, p_{x}\right)=\left(\sqrt{2}(s+r-1), \frac{2(s-r)}{\sqrt{2}}\right)
\end{aligned}
$$


By appropriate definition of the inner product of states [44], one can pass to the Minkowskian formalism by analytic continuation. Not all the discrete states found in Euclidean black holes can be mapped into the Minkowski case. This is true only for the states with $m=\frac{n}{2}$, where $n$ is an integer. As is clear from (20), there are extra states in the coset model as compared to the Liouville theory. For instance, part of the $\tilde{D}^{ \pm}$corresponding to $\frac{3}{8}(2 s-4 r-1)=\frac{n}{2}$, with $n$ integer, exist in the Minkowskian black hole but not in the corresponding $c=1$ flat string theory. The screening operator $(r=s=0)$ exists only in the series $C$, which contains an integer subset of the discrete values of tachyon momenta that lead to poles in amplitudes [20]. In the context of the scattering of light particles off the Euclidean black holes, these discrete tachyonic values are interpreted [47] as bound states of the string tachyon mode with the black hole background. In the context of Minkowskian formalism such states are completely absorbed by the black hole. This can easily be seen by looking at the reflection coefficient $R(p)$ of the quantum-mechanical scattering process. It assumes the form 447]

$$
R(p)=\frac{\Gamma(1-i p) \Gamma^{2}\left(\frac{1}{2}+2 i p\right)}{\Gamma(1+i p) \Gamma^{2}\left(\frac{1}{2}+i p\right)}
$$

For $p=i \frac{n}{2}$, where $n$ is a positive integer, the $R$-coefficient vanishes. The fact that these values are purely imaginary is a consequence of the Lorentzian signature. In the Euclidean black hole case the corresponding momenta are positive integers or half integers. From a purely conformal-field-theoretic point of view one can consider now the OPE of vertex operators corresponding to these discrete values. Asymptotically in space-time this would imply factorisation of the scattering matrix to one of the discrete states. This interpretation can be carried out in the fully-curved black hole background if one assumes that, in an experiment scattering a massless particle off the black hole background, the absorbed light particle excites a given discrete massive mode of the black hole. The latter then decays by emitting particles whose number is restricted by the nature of the excited massive states. To see this one can repeat the steps of section 3, assuming that the momentum of the light particle takes one of the discrete values that lead to bound states. The net result for the selection rules is

$$
\begin{array}{r}
j=\frac{1}{3} m-1+\frac{1}{2}(N-2) \\
j \geq \frac{1}{4}(N-5)
\end{array}
$$

Notice the correspondence with the flat space selection rules (14) upon making the analogy

$$
\begin{aligned}
& 2 j_{S L(2, R)} \rightarrow j_{S U(2)}-1 \\
& \quad \frac{2}{3} m_{S L(2, R)} \rightarrow m_{S U(2)}
\end{aligned}
$$


However (23) should only be considered as a formal correspondence. The set of discrete states in coset theory is larger than that of flat $c=1$ strings, and hence there are always extra selection rules in the former model.

The above selection rules have been originally derived for the case of the Euclidean black hole. To pass into Minkowski formalism one notices the following. From the cohomological approach of Distler and Nelson 44] it becomes clear that the higherlevel discrete states in this string theory are characterised by real $j$. The usual passage from Euclidean to Lorentzian black holes is then given by the replacement $m \rightarrow i \mu$, where $\mu$ is defined by the diagonalisation of the generator of the $S O(1,1)$ subalgebra in the coset model $\frac{S L(2, R)}{S O(1,1)}$. For the discrete states $\mu$ is taken to be purely imaginary, $\mu=-i m$, so both selection rules (22) can be translated directly into the Minkowskian theory. Again, however, in view of the inequivalent spectra of the two theories the physical content of the (formally identical) selection rules changes.

The physical interpretation of these selection rules is now clear. They constitute a manifestation of the underlying $W$-symmetry structure of the theory. The non-trivial conserved charges that characterise the background (black hole) play an analogous rôle to topological charges in conventional local field theories, labelling not particles but rather vacuum sectors of the theory where the scattering of particles takes place. The key rôle of the $s$-wave dynamics and the selection rules are reminiscent of the Callan-Rubakov [14] process in scattering off a monopole. Further we recall that the instanton number in the standard electroweak model also leads to selection rules restricting the number as well as the flavour of emitted particles, for given incoming states [48]. In our case, since there is only one flavour of particle, the only restriction concerns the number with which the exchanged state can interact. In contrast to the local field theory example, however, here we have an infinite set of selection rules that differ for each internal state, and an infinity of topological (conserved) charges which label the internal degrees of freedom of the black hole. It is this feature that explains naturally the large statistical entropy of the latter, with the entropy being defined [7] as the loss of information for an observer at spatial infinity who, ignoring the higher excited black hole states, measures only the classical energy and charge of a non-rotating black hole. However, these can in principle be distinguished, for example, by measuring the number of particles the black holes emit when they decay. We discuss another class of distinguishing measurements in the next section.

\section{Aharonov-Bohm measurements}

We have argued previously that the discrete states appearing both in the flat space $c=1$ string and in the black hole coset model are singular gauge transformations (or physically equivalent representations thereof). This picture is consistent with that stemming from ordinary strings, where the various states are viewed as gauge 
particles 49]. In higher-dimensional string theories, the fact that stringy symmetries mix the various mass levels implies spontaneous breaking of these symmetries. In two dimensions spontaneous breaking cannot occur [50], so the gauge $W$-symmetries constitute a set of unbroken string gauge symmetries. The selection rules discussed above are a manifestation of this. In topologically non-trivial backgrounds, like the Schwarzschild black hole, these gauge symmetries lead to an infinity of non-trivial conserved charges. The association of the latter with discrete (non-propagating) delocalised string states implies their topological nature. Formally this can be seen as follows. At the level of the target-space effective action the coupling of the (gauge) string states with the conserved currents $J^{M \cdots}$ that characterise the string background can be represented generically as

$$
\int_{\text {space-time }} \sum_{s=\text { string-states }} A_{M \ldots}^{s} J_{s}^{M \ldots}
$$

In the spherically-symmetric case the integral over the four-dimensional space-time reduces to a two-dimensional integral. In that case, the string states $A$ are singular gauge transforms $d \Lambda$, and the currents $J$ are induced by the world-sheet currents leading to the charges (5). From current conservation it follows immediately that the terms (24) are purely surface terms, and therefore they can only carry global (topological) information about the space-time manifold. This is consistent with the fact that these charges are exactly conserved in highly-curved space-times. In fact it is the only possibility.

The pure gauge nature of the string states that couple to the conserved quantum numbers of the black hole leads to a better analogy with ordinary Aharonov-Bohm experiments used to measure conventional discrete gauge hair in local field theories [51]. Take, for instance, the antisymmetric tensor hair in string-inspired black-hole theories involving gauge fields that couple to the antisymmetric tensor field strength. There both the gauge field strength and the antisymmetric tensor field strength are identically zero outside the horizon. Also the charge corresponding to the antisymmetric tensor field strength is a surface term [51] $\int_{S(V)} B$, over a two-dimensional surface $S(V)$ surrounding a three-dimensional spatial volume $V$. A similar thing happens here. The field strengths corresponding to the string states vanish outside the horizon, since the states are pure gauge transforms, with singularities at the origin of the black hole. The corresponding charges, characterising the black hole solutions, are also surface terms. Due to spherical symmetry, the charges are just conserved numbers, since surface terms in effectively two-dimensional spaces consist just of points. The surface character of the charges can be easily exhibited for the first few of them (e.g. the total energy of the black hole [27, 4]). That these charges characterise the spatially-asymptotic form of the coset model is another manifestation of this fact.

Having established these properties of $W$-hair, we now describe a gedanken experiment for measuring it. The process will be similar to that of antisymmetric 
tensor hair [51], but with some crucial differences. Consider the scattering of fourdimensional fundamental strings off a spherically-symmetric black hole background. One considers an interference experiment at a final point $B$ between fundamental strings that have travelled from an initial point $A$ via two paths: in flat space, and in a black hole environment. The world-sheet of the latter fundamental string encloses the black hole singularity at a point $C$, and this produces a surface-like coupling with the correponding charges of the black hole of the form (24). The latter being topological will only manifest itself as an Aharonov-Bohm phase in the wave function of the fundamental string, which can be measured via an appropriate interference experiment at the point $B$. Due to the fact that the charges in the black hole background are in correspondence [22] with the higher-spin states, in order to measure individual charges one has to use polarised fundamental strings where the particular string mode is excited. Here comes an important difference from ordinary strings. If we were living strictly in two dimensions, these modes could not propagate, due to their topological nature, so scattering these modes off the black hole is not possible in the ordinary sense. However, in four-dimensional topologically non-trivial spherically-symmetric space-times (whose singularity structure is described by two-dimensional strings [7]) one can consider propagating higher-level string states, whose $s$-wave sector resembles that of two-dimensional strings, symmetrywise. In this sense one considers a fundamental string whose world-sheet encloses a black hole at $C$. The fundamental string is polarised so that a level $N$ state is excited, say. The $s$-wave sector of these states (which is topological) couples to the conserved charges of the spherically-symmetric black hole and makes a non-trivial phase contribution to the wave function of the propagating four-dimensional string at $B$.

A final comment concerns the formal character of the process. For the string propagating in a flat space-time background . one can use the $c=1$ string theory to describe its propagation. However, in this case there will be a mismatch when one couples the two theories at $C$, due to the extra states that the coset model (black hole background) appears to have 44. Hence the most correct treatment, consistent from a string field theory point of view, would be to treat the flat spacetime as an asymptotic region of the black hole space-time. However, in view of the similarity of the target space symmetries between the two models, it is possible that the non-trivial conserved charges are in correspondence with the set of states of the asymptotic $c=1$ Liouville matter system. The existence of stringy symmetries in the coset model mixing levels and mapping, for instance, the discrete states $\tilde{D}^{ \pm} \rightarrow$ $D^{\mp}$ 44 offers support to these arguments, and probably implies that the actual (physical) spectrum of the coset model might be smaller than it appears. We hope to return to these important issues in a forthcoming publication [46]. 


\section{Conclusions and prospects}

We have argued [4, 5] that the infinite set of of gauge symmetries possessed by string theory maintains quantum coherence in black hole physics, which is impossible with the finite set of gauge symmetries present in any local field theory. Specifically, in the case of spherically-symmetric four-dimensional string black holes, which can be modelled using an effective two-dimensional string theory, we have argued that there are an inifinite set of stringy conserved charges, $W$-hair, associated with a phase-space-conserving $W$-symmetry, that maintain quantum coherence. In particular, we have shown that string black holes decay in the same quantum-mechanical way as conventional massive string states and that a thermal or mixed description is not necessary [6]. In this paper, we have shown how $W$-symmetry enforces selection rules for particle production in the scattering of a light particle off a black hole, reminiscent of particle scattering off a skyrmion or a monopole, and made the point that all the $W$-charges (which are topological in nature) can in principle be measured by Aharonov-Bohm phase interference experiments for suitable selected massive flat-space string modes. Thus $W$-hair is in principle measurable.

Several more-or-less technical issues, some very important and non-trivial, remain to be clarified. These include a complete characterisation of the symmetries of string theory in target space-time, and an explicit space-time representation of the $W$-symmetries which we have argued to be so important. These $W$-symmetries arise both in the black hole coset WZ model and in the $c=1$ flat-space model that corresponds to it at radial (spatial) and temporal (in perturbation theory) infinity. However, there are unresolved questions about the size of the coset model spectrum and its relation to that of the $c=1$ model. Moreover, we should emphasize that although we believe that the $s$-wave part of the full four-dimensional problem captures the essence and is now solved, and we have applied our arguments also to axi-symmetric four-dimensional black holes [7], we still attach great importance to proving that quantum coherence is maintained for generic four-dimensional spaces with event horizons and singularities.

However, we attach even greater importance to a more fundamental limitation of our analysis, namely that it applies in a given black hole background, and can be extended to discuss the evolution of a black hole towards the flat-space $c=1$ model that describes its end-point in perturbation theory, but we cannot yet describe non-perturbative transitions between string vacua, and hence we cannot yet be sure that they also respect quantum coherence. To establish the maintenance of quantum coherence also during tunnelling processes would require the extension of the infinite set of stringy gauge symmetries off-shell. We are optimistic that this can be done, since there is no apparent problem for quantum mechanics in the $c=1$ string field theory of Das and Jevicki [2], nor for the $c=1$ matrix model that is fully non-perturbative. However, a more complete understanding of non-perturbative phenomena in string theory is necessary for the discussion of relevant issues in black 
hole physics. We know that in perturbation theory a string black hole loses massenergy until it resembles a discrete state in $c=1$ model, but then what? The fate of a black hole is essentially non-perturbative. 


\section{Acknowledgements}

One of us (J.E.) thanks the Cosmic Ray Group of the Physics Department of the Universidade Estadual de Campinas for its hospitality during part of this work. N.E.M. would like to thank HARC (Houston) and the Theory Group of Los Alamos National Lab. for hospitality during the initial stages of this work, and D.V.N. would like to thank the Physics Group of the Rockefeller University for warm hospitality and discussions while this work was in preparation. The work of D.V.N. is partially supported by DOE grant DE-FG05-91-ER-40633.

\section{References}

[1] S. Mandelstam, University of Berkeley preprint, UCB-PTH-91/53 (1991).

[2] S. Das and A. Jevicki, Mod. Phys. Lett. A5 (1990), 1639.

[3] S. Mukherji, S. Mukhi and A. Sen, Tata Inst. preprint TIFR-TH-91-28 (1991).

[4] J. Ellis, N.E. Mavromatos and D.V. Nanopoulos, Phys. Lett. B267 (1991), 465.

[5] J. Ellis, N.E. Mavromatos and D.V. Nanopoulos, Phys. Lett. B272 (1991), 261.

[6] J. Ellis, N.E. Mavromatos and D.V. Nanopoulos, CERN-Texas A \& M preprint, CERN-TH.6309/91;ACT-53;CTP-TAMU-90/91 (1991).

[7] J. Ellis, N.E. Mavromatos and D.V. Nanopoulos, CERN-Texas A \& M preprint, CERN-TH.6351/91;ACT-55;CPT-TAMU-100/91 (1991).

[8] S. Hawking, Comm. Math. Phys. 43 (1975), 199 ; ibid 87 (1982), 395.

[9] S. Hawking, D. Page and C. Pope, Nucl. Phys. B170 (1980), 283.

[10] J.D. Bekenstein, Phys. Rev. D12 (1975), 3077.

[11] For a recent review see: I.J.R. Aitchison, Univ. of Oxford preprint OUTP-24P88 (1988).

[12] M. Karliner and M.P. Mattis, Phys. Rev. Lett. 56 (1986), 428; Phys. Rev. D34 (1986), 1991.

[13] E. Braaten, Phys. Rev. D37 (1988) 2026;

E. Braaten and M. P. Mattis, Phys. Rev. D39 (1989), 2737. 
[14] C. Callan, Phys. Rev. D25 (1982), 2141.

V. Rubakov, Nucl. Phys. B203 (1982), 311;

V. Rubakov and M.S. Serebryakov, Nucl. Phys. B237 (1984), 239.

[15] I. Klebanov and A. Polyakov, Mod. Phys. Lett. A6 (1991), 3273;

A. Polyakov, Princeton Univ. preprint PUPT-1289 (1991).

[16] J. Avan and A. Jevicki, Phys. Lett. B266 (1991), 35.

[17] G. Veneziano, Phys. Lett. B167 (1985), 388;

J. Maharana and G. Veneziano, Nucl. Phys. B283 (1987), 126.

[18] A. Sengupta and S. Wadia, Int. J. Mod. Phys. A6 (1991), 1961;

D.J. Gross and I. Klebanov, Nucl. Phys. B352 (1991), 671;

D.J. Gross, I. Klebanov and M.J. Newman, Nucl. Phys. B350 (1991), 621.

[19] K. Demeterfi, A. Jevicki and J. Rodrigues, Nucl. Phys. B365 (1991), 499.

[20] A.M. Polyakov, Mod. Phys. Lett. A6 (1991), 635.

[21] N. Sakai and Y. Tanii, Tokyo Inst. of Technology and Univ. of Saitama preprint TIT/HEP-173;STUPP-91-120 (1991);

D. Minic and Z. Yang, Texas-Austin Univ. preprint UTTG-23-91 (1991).

[22] G. Moore and N. Seiberg, Rutgers preprint RU-91-29, YCTP-P19-91 (1991).

[23] M. Evans and B. Ovrut, Phys. Rev. D39 (1989), 3016; ibid D41 (1990), 3149.

[24] E. Witten, Institute for Advanced Study, Princeton preprint IASSNS-HEP91/51 (1991).

[25] R. Brustein and S.P. de Alwis, Phys. Lett. B272 (1991), 285.

[26] G. Mandal, A. Sengupta and S. Wadia, Institute for Advanced Study, Princeton preprint IASSNS-10-91 (1991).

[27] E. Witten, Phys. Rev. D44 (1991), 314.

[28] D. Gepner, Nucl. Phys. B290 (1987), 10.

[29] M. Freeman, C. Pope, M. Sohnius and K. Stelle, . Phys. Lett. B178 (1986), 199.

[30] C.A. Lutken and G.G. Ross, Phys. Lett. B214 (1988), 357.

[31] I. Antoniadis, C. Bachas, J. Ellis and D.V. Nanopoulos, Phys. Lett. B211 (1988), 393; Nucl. Phys. B328 (1989), 117. 
[32] J. Ellis, J.S. Hagelin, D.V. Nanopoulos and M. Srednicki, Nucl. Phys. B241 (1984), 381.

[33] L. Dixon, J. Lykken and M. Peskin, Nucl. Phys. B325 (1989), 329.

[34] J. Lykken, Nucl. Phys. B313 (1989), 473.

[35] I. Bakas and E. Kiritsis, Berkeley preprint UCB-PTH-91/44; LBL-31213;UMDPP-92-37 (1991).

[36] F. Yu and Y.S. Wu, Univ. of Utah preprint, UU-HEP-91/19 (1991).

[37] F. Yu and Y.S. Wu, Univ. Utah preprints UU-HEP-91/01 and UU-HEP-91/09 (1991).

[38] I. Bakas, Phys. Lett. B228 (1989) 57; Comm. Math. Phys. 134 (1990), 487.

[39] M. Evans and I. Giannakis, Phys. Rev. D44 (1991), 2467.

[40] A.A. Tseytlin, Nucl. Phys. B294 (1987), 383.

[41] J. Polchinski, Nucl. Phys. B357 (1991), 241.

[42] J. Polchinski, Nucl. Phys. B346 (1990), 253.

[43] N. E. Mavromatos and J. L. Miramontes, Mod. Phys. Lett. A4 (1989), 1847;

E. D’ Hoker and P. Kurzepa, Mod. Phys. Lett. A5 (1990), 1411.

[44] J. Distler and P. Nelson, Univ. of Pennsylvania and Princeton preprint UPR0462T/PUPT-1262 (1991).

[45] D. Kutasov, E. Martinec and N. Seiberg, Princton and Rutgers Univ. preprint, PUPT-1293/RU-91-49 (1991);

G. Moore, M. Plesser and S. Ramgoolam, Yale preprint YCTP-P35-91;

S. Das, A. Dhar and G. Mandal, Inst. for Advanced Study-Princeton and Tata Inst. preprint, IASSNS-HEP-91/79;TIFR-TH-91/57.

[46] J. Ellis, N. E. Mavromatos and D.V. Nanopoulos, in progress.

[47] R. Dijkraaf, H. Verlinde and E. Verlinde, Princeton preprint PUPT-1252; IASSNS-22-91 (1991).

[48] G. 't Hooft, Phys. Rev. Lett. 37 (1976), 8; Phys. Rev. D14 (1976), 3432; Phys. Rev. D18 (1978), 2199;

[49] D. Gross, Phys. Rev. Lett. 60 (1988), 1229;

D. Gross and P. Mende, Nucl. Phys. B303 (1988), 407. 
[50] N.D. Mermin and H. Wagner, Phys. Rev. Lett. 22 (1966), 1133;

S. Coleman, Comm. Math. Phys. 31 (1973), 253.

[51] M.J. Bowick, S.B. Giddings, J.A. Harvey and A. Strominger, Phys. Rev. Lett. 61 (1988), 2823;

T.J. Allen, M.J. Bowick and A. Lahiri, Phys. Lett. B237 (1990), 47. 